
internationales

vol. $29-n^{\circ} 1 \mid 2013$

Mémoires et migrations en Afrique de l'Ouest et en

France

\title{
«Comment décrire ? Comment raconter ?... ». La mémoire perdue des hôtels meublés parisiens
}

"How to Describe? How to Tell?...". The Parisian Furnished Hotels' Lost Memory

«¿Cómo describir? ¿Cómo contar?...». La memoria perdida de los hoteles

amueblados parisinos

\section{Claire Lévy-Vroelant}

\section{(2) OpenEdition}

Journals

Édition électronique

URL : https://journals.openedition.org/remi/6295

DOI : 10.4000/remi.6295

ISSN : $1777-5418$

Éditeur

Université de Poitiers

Édition imprimée

Date de publication : 1 mars 2013

Pagination : 91-111

ISBN : 979-10-90426-07-8

ISSN : 0765-0752

Référence électronique

Claire Lévy-Vroelant, « « Comment décrire ? Comment raconter ?... ». La mémoire perdue des hôtels meublés parisiens ", Revue européenne des migrations internationales [En ligne], vol. $29-n^{\circ} 1$ | 2013, mis en ligne le 01 mars 2016, consulté le 14 avril 2022. URL : http://journals.openedition.org/remi/6295 ; DOI : https://doi.org/10.4000/remi.6295 


\section{« Comment décrire ? Comment raconter ?... ".. La mémoire perdue des hôtels meublés parisiens}

\section{Claire Lévy-Vroelant ${ }^{1}$}

\section{Introduction}

L'hôtel meublé est emblématique du logement des migrants venus de province ou du Maghreb (Faure et Lévy-Vroelant, 2007). L'entrée dans les lieux par la mémoire relève de l'expérience ordinaire tant le double corps des hôtels (Bégout, 2003) est marqué par les évènements, guerre d'Algérie tout particulièrement, et par le passage du temps. "L'émigration a aussi, dans les hôtels, les cafés et les chambres de service, ses lieux de mémoire " (Harbi, 2004 : 50). Ce secteur meublé, composé des garnis et des chambres d'hôtels, est encore occupé majoritairement par ceux qui furent les travailleurs immigrés, la plupart venus d'Algérie dès avant l'indépendance. La volonté de ne pas dépendre d'un patron, la facilité à acheter une licence, I'abondance d'une clientèle de compatriotes avait permis la constitution d'un secteur commercial en grande partie algérien, et particulièrement kabyle. Entre 2007 et $2010^{2}$ nous avons mené I'enquête dans une vingtaine de ces hôtels situés dans les quartiers populaires et mélangés de la capitale, le 11 ème (quartier Voltaire), le 18 ème (la Chapelle, Barbès, Clignancourt), le 19ème (Stalingrad) et le 20ème (Belleville, Couronnes), où se côtoient Maghrébins vieillissants, anciens légionnaires français, soldats au long cours issus des ex-colonies, immigrés plus récents de provenances diversifiées, personnes en rupture ou en transit (Barrere et Lévy-Vroelant, 2012).

Pourtant, dès l'abord, les cadres se dérobent : cadres sociaux instables et marginalisés, cadre physique mis à mal par le défaut d'entretien et la modestie des matériaux. Les opérations de rénovation urbaine et la spéculation immobilière ont largement contribué à réduire ce secteur réfractaire à la patrimonialisation : 20000 établissements à la fin des années 1920, environ 900 aujourd'hui.

1 Professeure de sociologie à I'université de Paris 8 Saint-Denis, Centre de recherche sur I'Habitat, UMR LAVUE 7218 ; clevyvroelant@gmail.com

2 Dans le cadre du programme " Mémoire de l'immigration, vers un processus de patrimonialisation ? " du Ministère de la Culture et de la communication, direction de I'Architecture et du Patrimoine. 
L'originalité de l'habité de l'hôtel se fixe sur les espaces du caché et du secret (les caves, les toits), et sur ceux de l'ouverture et de l'accueil (le café - qui " donne " sur la rue -, l'établissement, les chambres). Le lieu traceur devient le prétexte et le contexte d'un inventaire par l'absence, d'une activité mémorielle en creux dont les limites sont en relation directe avec la position subalternisée du groupe et des personnes qui le composent. Oralité des entretiens, écritures littéraires, c'est par la mise en mots, au présent du récit, que l'inventaire est possible. Nous avons fait dialoguer un corpus de textes littéraires, sélectionnés grâce à l'activation des mots-clés (chambre d'hôtel, hôtel meublé) dans une base de données riche de 3600 titres $^{3}$, avec les récits recueillis dans et autour des hôtels. Mais dans les textes littéraires comme dans les entretiens, c'est souvent le déni de mémoire qui sert d'amorce au récit du sujet par lui-même : "L'histoire de ma famille? C'est un sujet que personne n'évoque, comme si nous en avions honte, comme si le fait de secouer cette cendre allait nous porter malheur [...] Est-ce au métèque de faire office d'historien ou de griot de la société qui les accueille ?" (Lopès, 1997 : 24). L'immigration ou le tragique de l'écriture : " car espace sousdécrit, l'espace des immigrés est longtemps resté un espace sans parole. Bien plus : un espace qui exclut la parole "(Bonn, 1998 : 50).

Plus généralement, le lien entre la mémoire et la ville, tissé par ceux qui I'ont habitée, et retravaillé par les rapports entre les individus et les groupes, a donné lieu à des recherches consistantes et diversifiées (Hayden, 1994 ; Massey, 1995 ; Yong-Sook et Brenda, 2004 ; Veschambres, 2008). Pourtant, les lieux de vie et d'habitat des migrants demeurent pour la plupart dans l'obscurité du silence et de l'invisible. Sans qualités intrinsèques, ils semblent destinés à subir les opérations de rénovation urbaine insensibles à la valeur de ce dont ils témoignent. L'autre qui fait la ville, le migrant pourtant considérable ${ }^{4}, \mathrm{n}^{\prime} \mathrm{a}$ voix au chapitre que de façon intermittente, tronquée, incertaine. Reprenant la piste des traces-mémoires de l'écriture en pays dominé (Chamoiseau, 1997), et celle des subaltern studies (Spivak, 1988), nous avons travaillé I'hypothèse selon laquelle les voix inaudibles des subalternes et des colonisés (Mbmembe, 2006 : 117-133) avaient une chance d'advenir, ou de se remembrer, à travers l'écriture littéraire susceptible de les compléter et de les re-présenter.

Mais quelle mémoire les hôtels meublés de Paris peuvent-ils porter ? Arrièrecours des guerres coloniales, habitat et lieu de vie stigmatisés de générations de migrants, ils sont aujourd'hui rattrapés par I'" urgence sociale ". L'entreprise réformatrice menée par la mairie de Paris s'est efforcée de les transformer (Jankel et Lévy-Vroelant, 2007), tandis que le manque chronique de logements les désigne paradoxalement comme quasi impropres à I'habitation. Travaillés dans leur corps physique par d'interminables travaux palliatifs, et dans leur corps social par le vieillissement et la paupérisation de leurs habitants, ils offrent néanmoins une résistance étonnante. C'est que l'impossibilité de l'éradication se nourrit d'un défaut quasi structurel de logement pour les pauvres

3 La base numérisée Frantext a été créée par le laboratoire de littérature Atilf de l'Université de Nancy 2. Le corpus associé est constitué de romans et nouvelles d'auteurs maghrébins et caribéens francophones, outre les auteurs français. Pour le détail de la méthode, voir Barrère et Lévy-Vroelant (2012).

4 Pour reprendre le titre du livre d'Isaac Joseph (1984) Le Passant considérable. Essai sur la dispersion de l'espace public, Librairie des Méridiens-Klincksieck. 
et les migrants ; mais c'est aussi que la mémoire dont les hôtels sont porteurs n'en a pas fini d'irriguer les narrations urbaines. "Comment décrire ? Comment raconter ? Comment regarder ? [...] Comment reconnaître ce lieu ? Restituer ce qu'il fut ? Comment lire ses traces ? (Pérec, 1994 : 40). Prendre l'hôtel meublé comme un espace matriciel, un palimpseste embrayeur d'imaginaire, comme le principe organisateur d'un réseau de traces et d'empreintes : tel est le parti que nous avons pris, contre, il faut bien le dire, les représentations dominantes et parfois les intéressés eux-mêmes. Alors même qu'ils revendiquent la banalité des lieux et des existences qui y ont pris place, les récits forment une matière riche en mémoire. II ne s'agit pas, on l'aura compris, d'établir des " faits historiques ". Mais par la mise en lumière des régimes et des usages de la mémoire au présent, et du fondement mythologique sur lequel elle se construit, la signification historique de ces lieux se dévoile, la contribution de I' "immigration " aux cultures urbaines apparaît.

Dans la première partie, c'est la matérialité des hôtels qui nous intéresse, dans son rapport avec la mémoire. De la rue à la chambre, du café aux courettes intérieures, de la cave aux soupentes, une série de seuils orientent les usages: autant de lieux dans lesquels et à propos desquels le récit prend forme. Les textes littéraires et les témoignages recueillis au fil des entretiens offrent des structures communes. Les récits de nos interviewés, exprimés le plus souvent au " je " de la première personne, font corps avec les espaces; il n'en va pas autrement de la littérature qui s'écrit sur et dans les hôtels, et les prolonge. Ellipses, décrochages, reprises, rythment les récits mémoriels qui s'écrivent et se disent en fragments, en monologues, en conversations éclatées, en invectives à la cantonade ou en récits enchâssés d'une enquête qui met la mémoire, avec ses absences, en intrigue. Les lieux (le lieu d'où l'on parle, le lieu dont on parle) leur procurent appui et consistance.

Sans collectif porteur, point de mémoire collective - et une histoire plus que discrète, en marge de ses déclinaisons hégémoniques. La deuxième partie traite de ce qui fait tenir le groupe, par-delà les affiliations antérieures. Si les récits prennent appui sur les lieux, ils se conforment au collectif qui les entoure : I'hôtel est aussi un cadre social qui conditionne l'expression mémorielle. Sa forme est hétérodoxe, il tient de la famille, de la communauté, du voisinage, de la simple collectivité de hasard. Le régime des hôtels n'est pas celui des foyers. L'opposition originelle tracée par Abdelmalek Sayad entre la communauté de I'hôtel (et du bidonville) et I'anomie du foyer (et du HLM) a sa part d'arbitraire (Sayad, 1980 : 89-103). Pourtant, elle met sur la voie d'une particularité de I'hôtel qui est aussi, peut-être, la condition de l'expression de la mémoire : une identité-relation (Glissant, 1990) plutôt qu'une identité nationale, ou d'origine, ou de religion. Le rôle de l'hôtelier, plus souvent hôtelière, est crucial : protecteur ou dictateur, il fixe les règles, tolère, négocie, encadre, soutient, mais surtout contient. La mémoire opportuniste des clients s'adosse à la mémoire longue des hôteliers, mais notre hypothèse est qu'un fond commun les structure. Pour le décrire, nous prenons au sérieux l'hospitalité en ce qu'elle instaure un régime particulier, une autre forme d'économie (Schérer, 1993 ; Gotman, 2001), et mettons à profit la notion d'hétérotopie (Foucault, $1967: 46-49)$.

La troisième partie aborde la question de la transmission. Où s'en va la mémoire une fois racontée ? Par quelles voies/voix accède-t-elle, le cas échéant, 
à un statut collectif ? Qui témoigne des vies des migrants des hôtels ? Le maintien de l'exploitation commerciale n'est pas désiré par la génération des enfants en ascension sociale. Le travail historique semble découragé par la rareté des archives. Une certaine muséographie met en scène des traces qui ne sont parfois que de restes. Le marché immobilier tue dans I'œuf toute perspective de patrimonialisation ${ }^{5}$. Les porteurs potentiels de telles mémoires ne constituent pas, pour l'heure, des relais dont la légitimité serait établie et stabilisée. Les voies de l'énonciation par le sujet sont étroites, l'hôtel reste intouché par les " entrepreneurs de mémoire "(Pollak, 1993). La perte de la voix signe l'irrémédiable inégalité de position : " le monopole de la voix qui constitue le subalterne, le précaire ou l'exclu en personne invisible n'est tel que du point de vue de la voix elle-même qui s'arroge ce monopole et nullement du côté de toutes les voix disqualifiées. Celles-ci produisent des récits, des textes, mais ceux-ci se heurtent à la structure de domination qui les exclut [...]" (Le Blanc, 2010 : 7-14). Lorsqu'elle prête sa voix pour apposer le récit d'une expérience semblable, l'écriture, littéraire tout particulièrement, se fait relai, miroir, chambre d'écho. Participant d'une réécriture à partir du silence et de l'oubli, elle pourrait être aujourd'hui un des vecteurs les plus significatifs de la transmission d'une mémoire des hôtels et de ses anonymes par là même nommés. Enquête ethnographique et investigation littéraire sont donc, dans notre approche, intimement mêlées.

\section{Des lieux de mémoire et d'oubli}

Spatiale, sociale et corporelle, la mémoire s'incarne et se régénère dans des lieux, matériels ou symboliques, qui font sens et construisent une mémoire de la Nation (Nora, 1997), une mémoire collective (Halbwachs, 1994) ou encore une culture (Bhabha, 1994). Mais loin d'être univoque et consensuelle, la mémoire est plurielle et inégalement exprimée. Portée par des groupes qui n'ont pas la même consistance ni la même puissance d'expression, elle est une fonction du pouvoir (Halbwachs, 1997 ; Noiriel, 2007 ; Stora, 2007). Réciproquement, la ville et ses espaces perdent de leur substance sans leur passé et sans les traces de ceux qui les ont habités, manifestation partielle et partiale du passage des individus et des groupes. Gonflées et mises en scène pour la comédie urbaine (Hebbert, 2005), elles s'affichent pour témoigner d'un rapport de force favorable à ceux qui les promeuvent. Mais de par leur caractère aléatoire et parcellaire, elles parlent aussi de ce qui ne fait pas trace. Comme le suggère l'écrivain dans son interrogation fondatrice (Perec, 1978 et 1994 ; Modiano, 2005 et 2006 ; Semprun, 1994), l'effacement et le silence, loin de signifier une fin, sont au contraire au principe d'une réappropriation et d'une réinscription possibles. La mémoire collective est donc faite d'oublis, mais les lieux permettent que s'y accroche ce qui pourra faire trace, pour peu qu'un groupe en soit le porteur, et que les cadres sociaux en autorisent l'expression. Les récits, les narrations de la vie de tous les jours, sont de nature à réveiller les fantômes, et c'est dans les espaces habités que ceux-ci circulent.

\footnotetext{
5 À l'exception notable de I'Hôtel du nord, Quai de Jemmapes, dont le film de Marcel Carnet, sorti en 1938, a fait la réputation. II est devenu " un hôtel de tourisme mettant à profit la légende pour offrir aux riverains et aux étrangers venus respirer l'air de Paris une atmosphère chaleureuse et branchée, mais toujours fière de son passé [...]. II ne tient qu'à vous de venir écrire avec nous l'histoire de ce lieu de légende ", [en ligne] consulté le 01/06/2011. URL : http://www.hoteldunord.org/french.html
} 


\section{L'hôtel, un espace habité particulier}

L'hôtel meublé est avant tout un lieu d'habitat des migrants et des pauvres. Mais à la différence de l'habitat populaire ordinaire, du fait de l'exiguïté et la frugalité des chambres, les lieux sont rabattus les uns sur les autres, saturés d'usages. Précisément pour éviter le chaos toujours possible, du fait aussi que I'hôtel est un espace traversé et traversant, son organisation repose sur une hiérarchie subtile de seuils qui le rendent habitable. Chaque lieu, les chambres faisant à peine exception, possède une fonction de transition, et est placé sous un régime d'interactions particulier, fait de proximité et de distance, d'accueil et de défiance, mais où, toujours, la présence doit être négociée.

Dans tous les cas, on n'approche l'hôtel que par paliers. Sa présence sur la rue se repère par des signes plus ou moins discrets (enseigne, linge aux fenêtres, panonceaux indiquant les tarifs), mais l'accès au dedans, à la chambre, espace de repli, de récupération et de solitude, ne se fait pas directement.

Photo 1 : Dans le quartier de la Goutte d'or (Paris, 18 $\left.{ }^{\text {ème}}\right)$, l'hôtel Myrha porte le nom de la rue. L'annonce de se "loger au mois ou à la journée ", le numéro de téléphone bien lisible (sans son préfixe parisien cependant), le rideau de la loge indiquent, derrière l'apparente banalité et la modestie des lieux, une forme particulière d'hospitalité

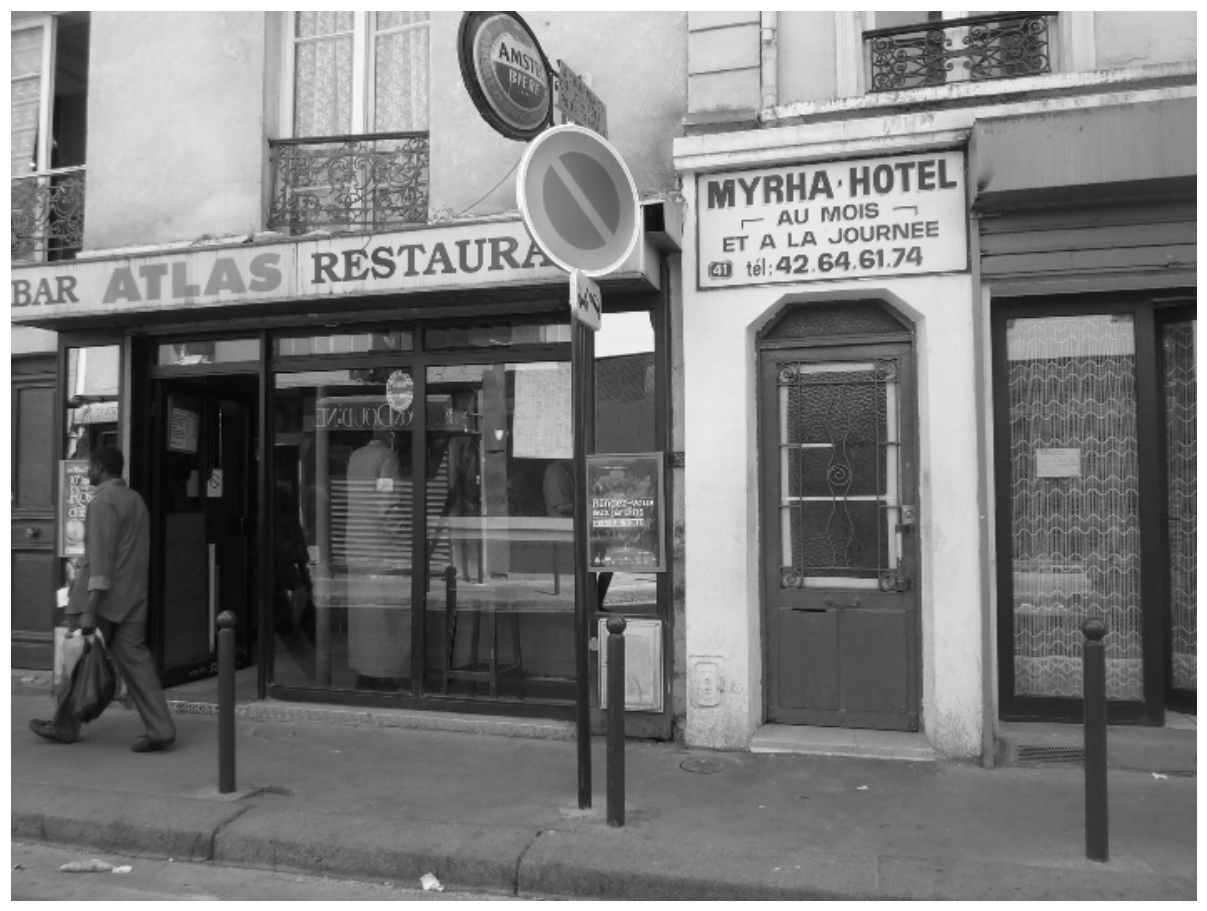

Source : Documentation personnelle de l'auteure. 
Entre la chambre et la rue, l'espace se diffracte en toutes sortes de lieux où I'hôtelier pose sa marque et surveille les conduites : pas-de-porte, café, courette, couloirs et paliers, ascenseurs et escaliers, cuisine, loge, caves et greniers. Les décorations évoquent souvent les lieux originels, et qui pourraient être communs : dessins ou photographies exposent des déserts de pierre ou de sable, des chameaux et des oueds asséchés, des jeunes filles en robes traditionnelles, des puits généreux. Survivant au passage du temps, divers objets signifient l'hôtel : un feutre d'homme resté accroché, des poteries décorées, des publicités de boissons, des photos anciennes, des fanions et des coupes. Ces objets revêtent, avec l'ambiance sonore (souvent dominée par la télévision) et olfactive, une importance capitale : éléments constitutifs de la vie dans les lieux, ils en adoucissent la promiscuité et l'inconfort. Les courettes, parfois passage obligé vers les toilettes, peuvent être l'objet de soins jardiniers. Accrochant le souvenir - tel hôtelier s'attendrit longuement sur le lilas rabougri qu'il a planté quarante ans auparavant - la matérialité des hôtels est prétexte à récits mémoriels. En littérature, elle entraine le souvenir de ceux qui l'ont habité. Ainsi des volets rouillés de l'hôtel où sont passés les parents de Dora Bruder, Boulevard d'Ornano, et de "tout un amas d'objets hétéroclites qui semblaient abandonnés là depuis longtemps " (Modiano, 2004 : 13-14).

II n'est pas indifférent que la chambre soit le lieu à partir duquel se construisent maints récits. Ceux-ci peuvent fonctionner en creux, comme pour I'homme aux cinquante chemises qui habite depuis plus de vingt ans, " un nid d'oiseau dans lequel il n'y a rien " (entretiens des 28 avril et 13 juin 2009), mais qui commence le récit du grand voyage (de Dakar) par l'évocation d'une petite valise en carton presque vide. Au contraire tel client fauteur de trouble met l'hôtel sens dessus dessous par l'usage abusif qu'il fait de sa chambre, la saturant de ses affaires au point que la porte ne peut plus s'ouvrir (entretien du 13 mai 2008). Mais laissé vide ou rempli à l'excès, l'espace physique de la chambre se dilate pour devenir l'espace d'une traversée de l'exil. II en retient le souvenir, abritant pour un temps le " moi valise " (Harel, 2005) de l'exilé au cœur de la métropole. En ce sens, I'hôtel est le réceptacle, le reliquaire de traces intimes, mais aussi la chambre d'écho d'un passé omniprésent, comme dans l'œuvre animée de I'artiste sud-africain William Kentridge (1994) Felix in Exile ${ }^{6}$. Celle-ci donne à voir l'expérience de l'exilé confronté, seul dans sa chambre d'hôtel, à la violence de l'arrachement, et de la perte des siens et de la femme aimée. Creusant le vide, la pièce se remplit de cette absence au moyen de feuillets volants qui, repeuplant la chambre de l'Afrique du Sud martyrisée par l'apartheid, viennent y inscrire les visages et les paysages perdus.

La chambre est cet espace matriciel où présent et passé sont comme condensés. Une fois la porte entr'ouverte, le dernier seuil est passé, et c'est le lit qui s'offre à la vue dès l'entrée : "On a vision sur le lit, en fait. II n'y a aucun filtre entre le public et le privé ", précise l'architecte chargé du diagnostic. La chambre est l'espace ramassé d'une intimité que le tenancier ou les voisins peuvent facilement troubler. La chambre est aussi l'espace de la vie et de la mort : le hasard d'un amour qui y donne la vie comme dans les souvenirs de petite fille cachée de Rachel (entretien du 2 février 2009), le hasard qui donne la mort, comme

6 Kentridge William (1994) Felix in exile. Geography of memory, [en ligne] consulté le 03/01/2011. URL : http://www.youtube.com/watch?v=vF5cngcXqSs 
dans nombre de récits d'hôteliers. Si la mort survenue dans la chambre hante ces derniers, c'est que, outre qu'elle les contraint à une vigilance de tous les instants, elle laisse l'enveloppe vide, au sens propre (du défunt, il ne restera plus que la trace du passage, les vêtements qu'il faudra débarrasser) comme au sens figuré (I'enveloppe est le mot employé pour signifier la dette de loyer laissée par le défunt, sans compter l'obligation de sacrifier aux usages de la collecte pour le rapatriement du corps). Espace du secret, du repli et du rêve, la chambre est enfin celui de la survie quotidienne : on y mange, on y dort, de cela on parle peu. La contrepartie implicite de la promiscuité est dans I'espoir que quelqu'un, dans les parages, remarquera une absence anormalement longue.

\section{Anecdotes et fragments}

Si la chambre et ses objets provoquent le souvenir, d'autres lieux de I'hôtel y sont aussi propices. Les caves et les toits, espaces du secret, enclenchent des récits de la guerre d'Algérie. Pour les plus jeunes, cette période lointaine est celle des gens cachés, traqués par la police, une période glorieuse auréolée de danger. L'évocation par tel hôtelier de réunions de cadres du FLN dans les caves de l'hôtel reste vague, il n'en a eu connaissance que par ouï-dire. Ce sont les anciens qui parfois racontent. Mais ceux-ci ne le font pas volontiers. Il suffit de dire que " ce café-là a une histoire de la révolution algérienne ". La page est devenue quasiment illisible.

Ce n'est que dans des circonstances particulières que le récit s'anime. Ici, il s'enclenche à la faveur de la lame de fond qui a saisi l'hôtel et dont nous sommes les témoins de passage : des travaux vont le transformer de fond en comble. On nous fait visiter. On raconte. À I'époque de la guerre, I'hôtelière était une toute jeune fille. Le frère blessé que l'on a caché sur le toit est le centre, le pivot et le cœur du récit, il conduit vers les autres, ceux qui ne sont jamais revenus. Sautant par-dessus un demi-siècle en une ellipse parfaite, l'évocation de ces hommes disparus l'amène à ceux d'aujourd'hui dont elle doit s'occuper. Le toit de I'hôtel a rendu un de ses secrets, l'absence fait lien, la justification de I'engagement de I'hôtelière tient dans ce rapprochement qui l'inscrit indéfectiblement dans la mémoire des lieux en même temps que dans l'ici et maintenant. " [...] Je me rappelle aussi de la manifestation d'octobre à Paris. 17 octobre 61 où les clients, la majorité, sont partis ; moi, je suis restée, mon frère a été blessé dans la manif ; quand il est revenu, je me rappelle, on l'a caché sur le toit, parce qu'après, ils ont fait des descentes dans les hôtels pour voir ceux qui étaient blessés, pour les arrêter. Ça veut dire qu'ils avaient participé à la manifestation. Puis certains qui ne sont pas revenus. Bon, je ne sais pas ce qu'ils sont devenus, mais il y en a qui ne sont pas revenus. Si, c'était... en fin de compte [silence]. Puis là, j'ai deux clients, qui sont là depuis très longtemps, je ne sais pas exactement combien de temps, j'ai jamais compris, enfin je ne sais pas, ils sont tout seuls, ils ne sont jamais repartis au pays [...]. Combien de fois je lui ai fait de la soupe, je lui ai fait monter ; K. va le voir s'il a des courses à faire, enfin, on est... que ce soit n'importe qui, hein. À partir du moment où ils ont une défaillance, soit malade, soit un problème, on est là, hein " (entretien du 14 mai 2009).

Le récit des hôtels se déroule en anecdotes singulières débouchant pourtant sur un horizon spatial et temporel élargi, à partir d'une adresse, d'un lieu, d'un signe, d'un objet : " ce sont des personnes qui laissent peu de traces derrière 
elles [...]. Ce que l'on sait d'elles se résume souvent à une simple adresse. Et cette précision topographique contraste avec ce que l'on ignorera toujours de leur vie - ce blanc, ce bloc d'inconnu et de silence "(Modiano, 1997 : 29-31). À partir des lieux traceurs de I'hôtel, les souvenirs s'épandent vers le dehors. Le Paris arabe des années de guerre, décrit en littérature (Poinçonnat, 2007), reprend forme, par exemple, au fil de la brève histoire de l'homme aux doigts coupés. Le matin même de notre passage, un jour de janvier 2011, un inconnu s'était arrêté le temps d'un café au comptoir. II avait alors livré cette histoire que I'hôtelier nous rapporte: "C'était les années de guerre, de rue en rue de Barbès à Clignancourt, des files invisibles d'hommes indiquaient avec des signaux de fumée, des cigarettes, quand la police se rapprochait ". Puis, poursuit l'hôtelier, I'homme avait pleuré et montré ses mains avec deux doigts coupés par les Harkis. Le 17 octobre 1961, il avait été jeté dans la Seine, et n'avait eu la vie sauve que parce que, par chance, il savait nager.

Remontant simultanément le temps et l'espace, la mémoire est activée, dans le récit comme dans l'écriture, par l'évocation des lieux où l'intrigue s'est nouée. Chambres, couloirs, toit, rue, élément du paysage : autant de repères dont la pérennité met en place une sorte de cadastre mémoriel où les vides importent autant que les pleins. Dans le premier extrait cité, l'hôtelière ne fait qu'évoquer brièvement l'épisode de la descente de police - mais nous aurons d'elle d'autres récits racontant les chambres mises à sac, les lits éventrés. Quant au récit de l'homme aux doigts coupés, il n'est rendu que par petites touches. Dans les deux cas, ce sont des lieux précis qui portent le souvenir. Parfois, c'est un fragment, une image hallucinée qui refait surface, comme dans le récit de cet ancien militaire, fils de Harki, dans la salle du café : "Moi, quand j'étais jeune, pendant la guerre d'Algérie, j'ai vu ils étouffaient les chiens, ils les pendaient... ! Ils pendaient les chiens pour pas qu'ils aboient la nuit ! Et quand vous allez à la rivière, maintenant, pour prendre un bain, vous trouvez des corps remplis d'eau... égorgés... vous croyez que c'est bon à savoir, tout ça ? ! II vaut mieux être à la morgue ! " (entretien du 13 juin 2009). À l'inverse des anecdotes morales mettant en scène des défunts turbulents, rapportées non sans plaisir par des hôteliers sur le ton de la confidence, les souvenirs de la guerre s'expriment à haute voix en fulgurances douloureuses. Mais I'hôtelier veille et contient, et le groupe des présents en atténue la portée mortifère.

\section{L'hôtel : portrait de groupe}

Les hôtels meublés, en particulier ceux qui ont un café, exposent en saynètes une humanité souvent âgée, mais composite et réactive : placides joueurs de dominos, exubérants complices de comptoir, buveuses de bière bavardes ou ouvriers taiseux se côtoient dans une apparente sérénité. Parfois, des éclats de voix se font entendre, ou bien l'ambiance se noie dans une tristesse épaisse. On est reçu par un hôtelier plus ou moins avenant, qui attend de voir ce qui vous amène. L'hôtel est un lieu d'ouverture. Parfois, la porte se referme sur une fin de non-recevoir. Lieu habité, l'hôtel est aussi une forme sociale complexe, dont les particularités ne sont pas pour rien dans les manières dont la mémoire s'exprime. 


\section{L'hôtel, une communauté ?}

Le régime des hôtels n'est pas celui des foyers. Mais peut-on pour autant parler de communauté ? Nos investigations montrent un monde contrasté qui ne se laisse pas saisir par les catégories habituelles. À l'origine, l'hôtel meublé accueillait, selon des processus semblables à ceux qui ont présidé à la constitution des bidonvilles, des immigrés originaires d'une même région et souvent unis par des liens de parenté. Aujourd'hui, on y retrouve des groupes d'interconnaissance ancienne dont les membres sont liés par des sentiments et soumis à des règles (solidarité, tolérance, discrétion) acceptées sans discussion. Mais une telle communauté, si tant est d'ailleurs qu'elle n'ait jamais existé sous une forme parfaitement homogène (Faure et Lévy-Vroelant, 2007 : 107-110), est nécessairement autre chose qu'un produit inchangé du passé. C'est dans le présent que se vit le groupe où dominent les restés sur place, dans une coprésence tributaire des conditions matérielles d'existence, mais aussi des attachements et des déprises par-delà le temps et l'espace. II existe une idée du groupe (Halbwachs, 1970 : 115) et c'est d'abord I'hôtelier qui en est le dépositaire et l'animateur. Si la guerre des mémoires n'a pas cours dans les hôtels, c'est que des formes de régulation puissantes sont à l'œuvre. Parmi les plus manifestes, celles qui ont pour objet la famille et les liens de parenté, et celles qui concernent la morale et les valeurs, comme nous allons le voir maintenant.

Les figures empruntant au registre de la parenté sont fréquentes dans les hôtels. Les mots signifiant une relation paternelle, maternelle, filiale ou fraternelle y abondent. L'image de la famille est mise en avant par des hôteliers, mais aussi par des clients. Pour les premiers, la famille est une réalité tangible et fondatrice de leur activité. Famille et hôtel se confondent même parfois tout à fait : le domicile privé est alors situé dans l'hôtel même, les enfants y ont grandi. L'Hôtel Familial, le bien nommé, a été le domicile privé de la famille de l'hôtelier pendant plus de trois décennies. L'hôtelière n'y habite plus, mais c'est avec son frère qu'elle tient l'établissement. Dans cet autre établissement, près de son père, travailleur immigré de la première heure, se tient le fils, titulaire d'une licence en sciences économiques. Mais, la familiarité s'étend à d'autres clients en alternance, les rôles circulent: "Ah oui pendant longtemps. C'était comme la famille. On rigolait... mon père, quand il sortait, il les laissait ici à la réception, quand il n'avait personne pour garder "(entretien du 11 mars 2009). Emblématique de ces hôtels achetés dans les années 1950 à leurs propriétaires français, celui de la familleT., originaire d'Algérie, propriétaire du fonds de commerce depuis 1977 et des murs depuis 1990. La gérance est passée du père au fils et du fils aîné à son frère cadet. Les chambres sont réservées, on s'arrange, on rend service, I'hôtelier appelle cela la " sécurité familiale " et revendique la dimension morale et humaine de son travail " parce qu'ils sont loin de leur famille et de leur pays ". Ce qui constitue un devoir sinon accepté, du moins reconnu : "chez les Berbères on est très communautaires [...] on n'a pas fait vingt-huit gosses, eh ben c'est comme si !" (entretien du 28 janvier 2009). Les liens de remplacement sont manifestes aussi du côté des locataires et ne jouent pas nécessairement sur la communauté d'origine. On loue le frère, celui en qui on peut placer sa confiance, tout en regrettant qu'un tel lien soit si rare. Tel client appelle I'hôtelière " ma fille ". L'image de la famille, cependant, est porteuse d'ambivalences. Les nouvelles circulent, on s'en réjouit autant qu'on s'en méfie. Le commérage met les personnes à l'épreuve, mais contribue aussi à renforcer la cohésion du 
groupe. "Il y a des gens qui viennent te dire, ah, tu sais, untel il a fait ça, il a fait ça, hier. Alors, si tu écoutes les gens qui disent des trucs comme ça! II y a des gens qui croient tout de suite ! Oh, il a fait ça... Or, c'est faux ! Vous voyez ce que je veux dire? ". Placer sa confiance en connaissance de cause, se méfier des rumeurs, mais au besoin en faire circuler soi-même : la vie à I'hôtel enseigne aussi à tenir ses distances. Quand tel jeune (fils) hôtelier évoque le terme de zaouia, forme de communauté impliquant des liens d'entraide engageant tout le groupe sous l'autorité spirituelle (religieuse) d'un chef, il suggère en même temps I'aura de son père, originaire du Maroc, et une forme de communauté élective : "Là, au mois de mars et avril, il y en a beaucoup qui vont revenir, parce qu'il y a des papiers à remplir, les impôts, tout ça. C'est comme ça, la zaouïa [...]. Si j'ai pris cet exemple de zaouïa par rapport à l'hôtel, c'est pour parler de ce côté lieu de vie. C'est-à-dire qu'il n'y a pas juste le caractère donner la clé de la chambre et monter, puis payer. Vous voyez, les clients, c'est des amis ". Dans une réciprocité qui rappelle un des fondements les plus anciens de I'hospitalité, I'hôte (I'hôtelier) et chacun des hôtes (les clients) sont engagés dans une relation qui en fait les obligés (certes inégaux) les uns des autres, dans la vie comme dans la mort : " mon père s'il devait aller en Algérie, à chaque ville où il irait, il a des amis. Parce que les clients, ils viennent de partout, d'Algérie. C'est des gens qui laissent leurs affaires ici, qui ont confiance. S'ils ont de l'argent, ils le laissent chez mon père, s'ils vont à l'hôpital, on va les visiter, voilà, donc. Et puis il y en a qui sont décédés ici, donc on s'est occupé de les ramener au Maroc" (entretien du 24 février 2009).

Une autre indication de l'existence d'une forme de groupe se rapporte à la morale et aux valeurs. La hiérarchie, propre à I'hôtel, des désirables et des indésirables, met en exergue le sens particulier accordé à l'argent, fortement socialisé dans un tel contexte. Clients comme hôteliers distinguent des figures: ainsi l'image du parasite colle aux " associations ", ces clients envoyés par les services sociaux, bien distingués des "travailleurs ". L'hôtel montre ses préférences. À ceux qui ont fini leur carrière (les retraités) ou à ceux qui, chômeurs, font preuve de bonne volonté dans la recherche d'un travail, on accorde respect et considération, mais aussi des aides ponctuelles en nature, voire un crédit : c'est ainsi que telle hôtelière laisse filer l'ardoise d'un jeune qu'elle veut soutenir, ou encore que des échanges de services et parfois d'argent s'effectuent entre clients. Les collectes pour le rapatriement des corps sont très fréquemment mentionnées. Finalement, il est deux sortes de clients dont le milieu des hôtels réprouve le comportement et qu'il voudrait exclure : les profiteurs dont le rapport au travail, à l'argent et à autrui, est corrompu. Plus l'hôtelier est engagé à faire prévaloir une gestion " humaine ", plus ce type de client le fragilise : " [...] et il emmerde tous les clients. Voilà [silence]. Donc, si par exemple [client Untel] me dépanne, il va dire, oui, je vais aller porter plainte, tu fais travailler au noir... Vous voyez, c'est des gens méchants, quoi. Il ne sait pas que si je ne suis pas là [client Untel] il dort dehors. Et entre [client Untel] et ce monsieur, c'est autre chose, quand même ! On est humains. Alors on va payer parce qu'on est humains ? ! I ne me paye pas[client Untel], il ne peut pas. II a juste pour manger. Il a une petite pension, il a une petite retraite. C'est par amitié, voilà. Et il bosse, il bosse. II m'aide. Voilà, on s'aide comme ça ".

Des personnes "vivant sur le dos de la société " sont d'autant plus méprisées qu'elles abusent non seulement de l'hospitalité de I'hôtel, mais de celle de la 
société tout entière. Une cliente prostituée n'est pas jugée en tant que telle, mais parce qu'elle affiche ouvertement ses préjugés contre les Arabes. Les mauvais payeurs peuvent racheter leur faute, les alcooliques se faire accepter, mais un comportement raciste est réprouvé. L'unanimité se fait autour de la dénonciation de la quintessence du mauvais client : mauvais payeur, mais surtout dénonciateur, raciste, égoïste et profiteur. " Je veux dire, nous, ici, on se serre les coudes, des fois, si tu n'as pas de sous, t'as faim, ben viens manger avec nous, machin. Mais lui, c'est des mecs, rien " (entretien du 14 janvier 2009). Les valeurs mobilisées puisent donc largement dans celles du monde ouvrier. Dans les années 1950, Andrée Michel avait mis I'accent sur la contestation radicale de la morale petite-bourgeoise par les familles ouvrières habitant I'hôtel (Michel, 1960 : 727-728). Doit-on voir dans la fierté d'avoir logé des personnalités hors du commun, fussent-elles déviantes, une manifestation de cet anticonformisme caractéristique du milieu ? Ici on se targue d'avoir eu quelques célébrités de la bohème, et des figures hautes en couleur, "des stars, mais pas des vraies stars, des stars entre guillemets... des gens qui passent toute sa vie dans les hôtels, alors... ". Faux monnayeurs, artistes en déroute, exilés silencieux, légionnaires poètes : autant de figures que I'hôtel ne renie pas.

C'est sans doute dans la façon dont l'argent est évoqué, dans ce milieu où il manque, que le renversement des valeurs en vigueur est le plus manifeste. L'argent y a une signification souvent unique dans l'équivalence qui en est donnée : le prix du billet payé par la famille à L., jeune émigrant, 540 francs, c'est "le prix d'une maison " et celui de sa dette. Mais bien utilisé, il assure la survie de l'homme et de tous ceux qui en dépendent: le calcul des dépenses de nourriture au centime près permet de se maintenir en bonne santé tout en continuant à assurer la subsistance des enfants. Le temps est alors argent socialisé : "I/s préfèrent rester ici deux heures, à jouer, pour 2 euros, que de sortir quelque part. Seulement consommer un café sur le boulevard, ça vous revient à 1,70 euro. Et un café c'est cinq minutes. Là, ils sont dans le chaud. En même temps, ils font passer le temps" (15 janvier 2009).

Les façons dont l'argent se gagne et se perd engagent les relations individuelles et lient l'accueillant et l'accueilli en un système d'obligations qui les précède et leur survit. La maitrise de l'argent contemporain, financiarisé et bancarisé, demande de toutes autres compétences. Les hôteliers ayant adopté une gestion rationalisée (et plus rémunératrice) à la journée revendiquent des relations neutres sur le modèle de la prestation. À l'inverse, l'hôtelier ancien style, qui ne peut s'affranchir du cycle du don et du contredon, est mis à l'épreuve de la difficulté à tenir le groupe. On nous a rapporté le cas d'un ancien hôtelier acculé à la faillite et à la rue, " parce qu'il ne payait plus ses loyers". L'histoire est exemplaire et la figure du " marchand de sommeil " un raccourci impropre.

\section{L'hypothèse de l'hétérotopie}

Ouverte sur l'extérieur et acceptant par la force des choses l'effraction de I'arrivant inattendu, la collectivité de l'hôtel meublé traditionnel s'oppose à celle des formes modernisées et asilaires, simple agrégation d'individus étiquetés " sociaux " qui se croisent et s'évitent dans les centres d'hébergement et les résidences sociales, où la mise à l'abri résulte des œuvres de l'État hébergeant 
(Lévy-Vroelant, 2000). Les idées du monde portées par l'une et par les autres diffèrent autant que leur morphologie sociale. La différence entre les hôtels et le reste des espaces sociaux, y compris les " maisons " peut être pensée sur un mode encore plus radical. Car les hôtels ont précisément « [...] la curieuse propriété d'être en rapport avec tous les autres emplacements, mais sur un mode tel qu'ils suspendent, neutralisent ou inversent l'ensemble des rapports qui se trouvent, par eux, désignés, reflétés ou réfléchis" (Foucault, 1984 : 46-49). Revenons à Céline: "L'hôtel, parlons-en, c'est plus inquiet, c'est pas prétentieux comme un appartement, on s'y sent moins coupable. La race des hommes n'est jamais tranquille et pour descendre au jugement dernier qui se passera dans la rue, évidemment qu'on est plus proche à l'hôtel. Ils peuvent y venir les anges à trompettes, on y sera les premiers nous, descendus de l'hôtel. [...] Tout peut se mettre à trembler de la terre au ciel d'un moment à l'autre, on est prêts, on s'en fout nous autres puisqu'on se "pardonne" déjà dix fois par jour rien qu'en se rencontrant dans les couloirs, à l'hôtel " (Céline, 1981 : 357-358).

Les hôtels sont à la fois hors de la société (par leur marginalisation, mais aussi leur potentiel de résistance et de déviance) et au cœur de cette dernière (faisceau de relations mondialisées et lieu d'entrecroisement de mémoires par-delà le temps). On y trouve des relations familiales hors famille, et des interprétations libres des valeurs en usage dans I'ordre marchand dominant. Ils sont des espaces à la fois ouverts et fermés, à I'hospitalité ambiguë et aussi des hétérochronies, non pas tant par leur caractère quelque peu anachronique, mais parce qu'ils se jouent des temps et des cycles de l'expérience sociale ordinaire : "Lui, c'est un ancien. Ça fait douze ans qu'il est là. Moi ça fait un an. J'ai divorcé et je me suis retrouvé ici. Mais je me demande ce que je fous là " (entretien du 13 mai 2008).

Vus de l'intérieur, les regroupements et les exclusions s'effectuent selon des idées qui, tout en empruntant au fond commun des cultures populaires (solidarité, valeur du travail, débrouille), n'épousent en rien les catégorisations officielles ou implicites : ni la langue, ni la nationalité, ni la religion, ni la couleur de la peau ne semblent influencer les relations sociales à I'hôtel. Immigré, étranger, cela même sonne faux : "Écoutez, pour moi, chacun qui a passé plus de cinq ans à Paris, il est Parisien [...] mon père il a un passeport étranger, mais il est depuis cinquante ans à Paris, alors! Mon grand-père il est depuis soixante ans à Paris... il est un étranger alors ? II a fait deux guerres pour la France... arrêtez !" (entretien du 14 mai 2008). Les ouvriers peu qualifiés et les petits employés exerçant leur métier y étaient la majorité, ce sont maintenant les chômeurs et les retraités qui sont les plus nombreux.

Les cohabitations privées génèrent des arrangements mémoriels quotidiens qui n'opposent pas les protagonistes selon leur histoire. C'est dans un présent de l'hôtel retravaillé par les injustices d'aujourd'hui et le sentiment d'une position sociale infériorisée que se coulent les souvenirs d'autrefois. Chacun protège sa vérité et fait parfois valoir sa vision du monde, ses fidélités : " C'est comme les anciens combattants. Un ancien combattant français, un ancien combattant indigène, ils n'ont pas les mêmes revenus [...]. La retraite c'est pareil [...]. Regardez mon mari, il était en France depuis l'âge de dix-huit ans, il a toujours travaillé, toujours cotisé ; ce n'est pas normal que sa pension, que sa retraite ne soit pas la même qu'un travailleur salarié français qui a fait 
les mêmes boulots ! [...]" explique cette hôtelière (entretien du 12 mai 2009). Mais les " étrangers " ne sont pas les seuls à parler de discrimination et d'injustice. Les hôtels comptent nombre d'anciens militaires, engagés ou appelés de l'armée française, également marqués par une expérience de domination, voire d'humiliation. "De Gaulle ? [...] II nous a jetés comme des merdes ", lance cet ancien d'Indochine et d'Algérie devant son hôtelière fille d'indépendantistes algériens (entretien du 28 avril 2009). La distinction entre Français et étrangers y perd de sa consistance, les identités se jouent des assignations collectives. Offrent-ils pour autant la possibilité d'une politique du semblable (Mbembe, 2006 : 120) ? II serait naïf de le prétendre (au vu ne serait-ce que de l'inégalité flagrante de position entre l'hôtelier et les clients), mais trop hâtif d'en repousser l'éventualité. Le régime d'hospitalité est " une économie, au sens total, car [il] rétablit sans cesse la production et la circulation des flux qui auraient tendance à s'immobiliser et à s'appauvrir " (Schérer, 1993 : 126). Dangereuse (car encline par nature aux abus), arbitraire (car l'accueil dépend du bon vouloir de l'hôtelier) et fondatrice (car le groupe est constitué d'hôtes, ceux qui reçoivent et ceux qui sont reçus), I'hospitalité participe de l'étrangeté de ces lieux hybrides, tenant de la famille sans en être, participant de réseaux sociaux mondialisés et néanmoins confinés, esquivant la marchandisation des relations et se jouant des normes prévalant tant dans I'habitat ordinaire que dans les centres d'hébergement ; toutes institutions sociales qu'ils interprètent à leur manière, participant ainsi d'une résistance au lissage et à la standardisation des cultures urbaines, mais aussi de la circulation (à bas bruit) de mémoires socialement et politiquement minorisées.

\section{La mémoire des hôtels au risque de la transmission}

La mise en regard de la littérature et des entretiens a permis de recueillir des mémoires in situ et d'envisager les hôtels comme des milieux de mémoires. Mais l'interrogation première (comment décrire ? comment raconter ?...) se prolonge par la question, plus lancinante encore, de la transmission. Où s'en va la mémoire une fois racontée, que deviennent les lieux une fois désaffectés, à quelles nouvelles narrations contribuent-ils ? Tandis que l'incertitude règne quant au devenir de la plupart de ces établissements, la muséographie naissante de la France des immigrations met en scène "le mobilier et les objets personnels laissés sur place [qui] deviennent aujourd'hui des supports pour retracer I'histoire et la mémoire de ce lieu $"{ }^{7}$. Mais détachées des relations sociales et des évènements qui leur donnaient sens, que disent encore ces traces ? Initiées au contraire par les héritiers reprenant à leur compte un désir de mémoire et de transmission, des associations se constituent pour porter le témoignage de ce que furent les vies de leurs parents ou grands-parents immigrés ${ }^{8}$. Si les voies et les voix de l'énonciation sont multiples, l'écriture littéraire demeure une forme majeure de l'expression mémorielle. II faut revenir sur les conditions du souvenir.

\footnotetext{
7 Guide de I'exposition permanente, pp. 130-131. II s'agit d'objets récupérés dans le foyer Rhin-et-Danube de Vaisse (Lyon) exposés au musée permanent de la Cité Nationale de I'Histoire de I'Immigration à Paris.

8 Nous n'abordons pas ici cette question. Voir par exemple Ahmed Boubeker et Adbelalli Hajjat (2008) Histoire politique des immigrations (post)coloniales, Éditions Amsterdam.
} 


\section{Passations, ou le coût du souvenir}

Se souvenir a un coût, mais il n'est pas le même pour tous. Dire " je me souviens ", c'est accepter de pactiser avec le temps qui passe, avec le temps qui est passé ; c'est renoncer à l'oubli et c'est, aussi, reprendre l'initiative (RiotSarcey, 2002). Chaque " je me souviens ", comme dans la liste de Georges Perec, témoigne et fait trace (Perec, 1978). À partir de cette énonciation, un pont est jeté, prenant appui sur le passé et réinscrivant le présent comme un nouveau point de départ. Faire advenir le souvenir procède d'une entreprise autobiographique, d'une réécriture de soi. Mais bien au-delà, il s'agit d'une affaire qui engage soi-même avec les autres, car comme l'écrit encore Perec : " ces "je me souviens" ne sont pas exactement des souvenirs, et surtout pas des souvenirs personnels, mais des petits morceaux de quotidien, des choses que, telle ou telle année, tous les gens d'un même âge ont vues, ont vécues, ont partagées, et qui ensuite ont disparu, ont été oubliées ; elles ne valaient pas la peine d'être mémorisées, elles ne méritaient pas de faire partie de l'Histoire, ni de figurer dans les Mémoires des hommes d'État, des alpinistes et des monstres sacrés. II arrive pourtant qu'elles reviennent, quelques années plus tard, intactes et minuscules, par hasard ou parce qu'on les a cherchées, un soir, entre amis ".

Cette explication donne une des clés de la nature de la mémoire exprimée : la possibilité de son émergence, surtout pas comme souvenir personnel (ce qui est aussi la proposition d'Halbwachs), mais néanmoins portée par un " je ", un sujet. Peu de nos interlocuteurs se sont permis cet exercice : signe que " ce que les gens de leur âge ont vécu " n'a pas lieu d'être évoqué, ou ne peut l'être pour cause de déchirure au sein même du groupe d'âge ; signe aussi que les familiers avec qui le souvenir pourrait prendre sens dans le présent du récit sont absents, disparus, ou ne recherchent pas cette connivence. De fait, les récits des hôtels sont faits de blancs et de manque, la perte de mémoire associée à l'impuissance et à des accidents dont on ne sait presque plus rien : "On m'a donné un coup de barre à mine. Alors ils m'ont emmené à l'hôpital. J'y suis resté quinze jours. J'étais complètement dans le coma. Qui c'est qui m'a frappé ? Je ne sais pas [...] La mémoire ? J'ai une courte mémoire, c'est tout. Tu as compris, maintenant ?" (entretien du 12 mai 2009). L'oubli peut même être volontaire, recherché, revendiqué : " J'essaie d'oublier... II ne faut pas remuer... comment on appelle... le purin. Qu'est-ce qui est le plus difficile dans le passé ? C'est toujours les douleurs qui remontent, de temps en temps. Si, par exemple, on vous écrase votre fille ou un de vos gamins, les souvenirs ils restent, on va rester traumatisé le restant de notre vie... Le traumatisme, c'est ça. [...] Je vais vous dire une chose, je préfère couper la conversation " (entretien du 13 février 2008).

Pourquoi se rappeler les souvenirs qui fâchent, cet héritage incorrect qui dérange ? La valorisation d'un passé à travers les lieux d'habitat et de travail est décidément une gageure, dont le caractère improbable est particulièrement manifeste dans les ateliers de mémoire pour habitants préconisés par l'Agence nationale de rénovation urbaine pour " accompagner " les démolitions et les déménagements qui s'ensuivent (Buscatto, 2006). Dans les hôtels, c'est aussi l'ambivalence qui caractérise le rapport aux lieux, et, partant, aux récits mémoriels susceptibles de $s^{\prime} y$ dérouler. Qu'elles soient légères ou tristes, qu'elles adoptent le ton du conte, de l'aveu, de la confidence, ou se coulent dans une conversation anodine, les évocations mémorielles sont volatiles, rarement 
revendicatives. La volonté de transmettre, de reconstituer, de remembrer (comme le mot anglais le laisse voir) n'implique-t-elle pas que l'on puisse aussi se caler sur l'histoire de sa génération ? D'anciens habitants reviennent en visiteurs, et certains quotidiennement, dans les hôtels qu'ils ont habités. Mais si le groupe des anciens de I'hôtel survit à la dispersion résidentielle, un tel cadre social est-il suffisant pour porter la mémoire?

Les souvenirs des hôteliers, déroulés dans la continuité de la transmission familiale, se disent plus facilement. L'achat premier, la clientèle et l'ambiance du café, la façon dont l'établissement était tenu, les passations successives, et même les conflits familiaux, constituent des repères assez facilement évoqués. Telle hôtelière, remontant le temps, se plait à décrire l'installation en France de ses parents venus d'Algérie juste après l'indépendance. Le récit des démêlés judiciaires avec l'actuel propriétaire des murs s'entrecroise aux moments traumatiques de son parcours. La figure paternelle domine encore les lieux. Autodidacte, il avait obtenu une licence de taxi, mais « ce parcours des immigrés en France n'est pas reconnu ". Elle parle du refus de la France, enchaîne sur son déplaisir de petite fille à habiter I'hôtel, qui était pourtant sa maison. Un moment particulièrement difficile est évoqué avec la peur de se retrouver à la rue. Mais avec son compagnon, elle a pu acheter un appartement en propre, et ainsi " épargner cette honte à ses filles ". La nécessité de contrer un mariage arrangé, le poids d'un destin familial brisé par des décès précoces, l'amour filial malgré tout, forment la trame d'un récit qui se joue de la chronologie et qui l'a vue " mourir et renaître en devenant quelqu'un d'autre " (entretien du 5 mai 2008). L'effort permanent pour " s'en sortir ", l'empilement des charges au quotidien, l'intrusion toujours possible d'un élément perturbateur engagent les conditions de l'expression. Le "je me souviens " des hôteliers passe par des récits mémoriels longs où évènements, faits et gestes du quotidien, et dimensions morales, sont solidairement enchevêtrés. L'assise que donne la propriété du fonds de commerce (plus rarement des murs) et la continuité familiale facilitent le récit. Pourtant, les enfants ne reprendront pas. Aussi, faute d'ancrage dans des lieux qui demeurent transitoires et éphémères, c'est par la mise en mots des valeurs revendiquées que la mémoire chemine et pourrait se transmettre.

\section{Ce qui vaut d'être transmis}

Un bien qui ne passe pas, ou rarement, mais des valeurs qui passent : les histoires familiales et les récits de la vie ordinaire usent des valeurs morales comme supports. La conviction que "ce qu'on vous a laissé ne vous appartient pas " témoigne de ce que l'argent est profondément marqué par l'obligation morale qui résulte de la transaction commerciale d'origine, et des devoirs qui la sous-tendent. De ce fait, le besoin de reconnaissance de leur travail hante les hôteliers. Les dons, les prêts, les attentions, sont mis en scène, commentés, justifiés et l'esprit qui les porte opposé à la perversion des valeurs qui menace dedans et dehors. On nous rapporte avec indignation que tel client, victime d'une crise d'épilepsie en pleine rue a été dépouillé de son téléphone portable et de son argent. Hier on était plus solidaire, " on allait taper chez le voisin, t'as pas une bouteille de lait, je te donne un paquet de beurre en échange... un paquet de cigarettes... ça n'a rien à voir, c'est plus la même relation... " (entretien du 5 mai 2008). Ces valeurs d'entraide sont revendiquées, enfin, contre l'alignement sur la richesse matérielle comme unique référence. Comme le dit un jeune 
client, ce qui l'intéresse, ce sont "les vraies gens ", ceux qui ont " de l'écoute " (entretien du 11 mars 2009). Or, même à l'hôtel, " souvent c'est chacun pour soi ". La tentation du repli est forte. "J'avais des problèmes financiers [...] à un moment donné, je te dis la vérité, j'ai failli... ma fenêtre donnait dans la cour, j'avais même rédigé un courrier, une lettre, comme ça, sur la table, je voulais me suicider ", nous confie un client (entretien du 28 avril 2009). La misère attaque I'hôtel au cœur, on comprend que chacun, même si le collectif encadre, se tienne sur la réserve. L'hôtelier doit s'en débrouiller, entre pragmatisme et charité. Commentant les photos d'un repas de l'Aïd, c'est par le montant de la dette laissée en partant que I'hôtelière désigne chacun de ses anciens clients : dette dont elle dit, non sans humour, qu'elle est un objet de fierté : " ma fille, elle me dit, je suis fière que tes clients te doivent de l'argent [...] parce [je ne suis] pas la patronne qui chasse. Ils sont conscients aussi qu'il y en a qui profitent de ma gentillesse. Mais ils sont contents, au contraire, surtout la dernière [...] ". À ses enfants, cette hôtelière nous dit avoir répété depuis l'enfance : "vous êtes les ambassadeurs de votre pays. À travers vous, on jugera votre communauté. On jugera l'Algérie [...] parce que c'est comme ça qu'on ne fait pas peur aux autres quand on est différent " (entretien du 14 janvier 2009). Le commerce, par nature, cherche à bonifier le chiffre d'affaires, mais fait abstraction des distinctions, classements et préférences. Demande-t-on d'où il vient ou qui il est, à celui qui se présente pour demander une chambre ? L'hospitalité, cette vertu interstitielle et dérangeante, renoue ici avec le religieux. Si le bien ne se transmet pas, le souvenir d'un accueil inconditionnel colore d'universalité l'altérité des hôtels, conférant à ce milieu une forme de permanence, et même d'éternité. Est-ce suffisant pour qu'ils passent à la postérité ?

\section{La mémoire du sujet, un enjeu politique}

Les récits des hôtels mettent en scène des mémoires en fragments, dont l'expression même est problématique, et ouvre sur l'impossible figuration de l'espace comme habitable par le sujet : c'est cela qui se dit en filigrane dans ces lieux d'énonciation précaire (Barrère, 2009). Cette impossibilité procède d'un double effacement, dont les démolitions et autres recompositions urbaines ne sont que le symptôme le plus visible : auto-effacement des sujets subalternisés, mais aussi naturalisation des mêmes sujets par les attentions, savantes ou non, dont ils sont l'objet. La faiblesse des cadres sociaux susceptibles de porter l'expression du souvenir transparaît dans les récits des hôtels. Le soutien, pour les clients, des " gens de leur âge ", n'est pas ou peu assuré, car les générations ont été désunies par la guerre et l'exil. Si un tel groupe générationnel parvient tout de même à se constituer à l'hôtel, il doit aussi composer avec les autres, qui ne sont pas non plus nécessairement ces amis avec lesquels, un soir, on se plait à évoquer le passé. Autour de la table, pour le jeu de domino quotidien, au comptoir, pour un café ou un verre, les choses du passé dont on pourrait sans risque évoquer le souvenir se sont évanouies.

Les habitants des hôtels ne sont pas des personnes remarquables, ils revendiquent même une insignifiance. Lorsqu'elles existent, les descriptions liminaires des hôtels se réduisent à un simple constat dépréciateur. Transmettre une mémoire de tels lieux en prenant appui sur l'édifice n'est pas d'actualité. II est presque impossible de les appréhender selon les principes de conservation et de protection classiques qui s'intéressent plus facilement aux édifices vides 
et à ceux qui ont abrité des personnes célèbres à un titre ou à un autre ${ }^{9}$. Si les lieux sont sans qualité et sans reconnaissance, faut-il imaginer une exportation de la mémoire hors les murs, penser un recueil organisé de témoignages ? Mais comment dépasser alors la simple accumulation de récits qui, faute de mise en valeur et de mise en contexte, pourrait les condamner à un oubli plus sûr encore ? La question des acteurs susceptibles de susciter et de porter la transmission, sous quelque forme que ce soit, est première. Or, les initiatives de groupes, qui pourraient en revendiquer l'héritage, se voient dans l'ensemble découragées par des politiques contraires, mais aussi par leurs propres trajectoires. Quant à la place occupée par les hôtels meublés dans la muséographie actuelle de l'immigration, elle est réduite à sa fonction de logement misérable, au mieux tremplin vers l'intégration dans le sein de la nation généreuse : " peu à peu on s'enracine ${ }^{10}$. $C^{\prime}$ est par le langage, par la voix du sujet parlant, que la jonction entre passé et présent pourrait s'opérer. Or ceux-ci restent, pour l'essentiel, muets. Cela n'est pas l'effet du temps qui passe. Leur voix a été recouverte par des narrations plus puissantes.

\section{Conclusion}

Traités comme des séquelles disgracieuses d'un âge révolu, les hôtels meublés sont en voie de disparition. Leurs photographies sépia décorent déjà les murs des cafés à la mode des quartiers renchéris, selon le processus qui consiste à mettre à profit les traces de culture populaire afin qu'elles participent à la valorisation de l'image des lieux, et finalement de la rente foncière et immobilière. La muséification, et particulièrement lorsqu'elle porte sur des sujets faibles, comporte aussi le risque de redoubler l'invisibilité des relations sociales qu'elle prétend montrer. Lieux de relations, assez indifférentes aux sommations identitaires, les mémoires des hôtels puisent à la condition ouvrière, militaire et subalternisée. C'est dans le télescopage des souvenirs, dans la confrontation incertaine avec ceux qui partagent le même lieu de vie que s'inscrit une expérience collective faite de singularités. L'hôtel, milieu de mémoires, a des histoires à transmettre. Des histoires de guerre et d'humiliation, des histoires de ruse et de manières de survivre. Mais comment témoigner et comment transmettre, quand les appuis légitimés manquent, les visions dévalorisantes installent un silence durable, et que les lieux eux-mêmes se dérobent?

Cette question invite à repenser le lien entre mémoire et histoire. Sans un arrimage solide à l'histoire des établissements et de leurs protagonistes, l'activité mémorielle est difficile et plus douloureuse. Pourtant, le jeu des imaginaires mis en place par et dans ces milieux de mémoire improbables peut constituer un contrepoids à une narration univoque et excluant le sujet, un espace et un support d'expressions mémorielles reconstituantes. Dans une telle perspective, l'objet n'est plus tant de saisir la place de l'immigration dans le récit national que de l'ouvrir à un récit autre qui en réfléchit les manques et ce faisant l'enrichit. Les

9 À l'inverse, le Tenement museum, situé à New York dans le quartier de Lower East Side de Manhattan, établi dans un ancien immeuble de rapport proche du garni et mettant en scène la vie d'une immigrée italienne, propose une forme de muséographie difficilement concevable en France, [en ligne] consulté le 20/06/2010. URL : http://www.tenement.org/

10 Guide de I'exposition permanente de la Cité Nationale de I'Histoire de I'Immigration, p. 119. 
textes littéraires participent de représentations plus généreusement partagées, plus hospitalières, plus créatives et plus critiques. Les récits de ville et d'errance migratoire, réarticulant temps et lieux librement, produisent du légendaire du fait qu'ils plongent dans les espaces irréguliers de la mémoire et de l'absence. Les descriptions qui en découlent proposent un ordonnancement du monde, une voie d'accès à la construction d'un sens. Instruisant l'expérience " dans ce qu'elle a de problématique " (Heinich, $2007: 36$ ), le milieu des hôtels remet en question la continuité linéaire des faits et des évènements et les guerres de mémoires trop hâtivement déclarées.

C'est là qu'intervient le rôle crucial de ce qui est énoncé. Partant du témoignage individuel, agglomérant plutôt que classant, la mise en mots, quelles qu'en soient les formes, propose un répertoire des états et des figures d'une recherche identitaire inachevée, car toujours en travail. Elle n'est pas chose finie, mais relation, un va-et-vient permanent entre le sujet et son milieu, bref, un trajet anthropologique. La mise en mots de la mémoire par le sujet fonde une expérience susceptible d'être transmise. Elle restitue une profondeur à la fois historique et sensible aux territoires urbains. Elle a pour alliée certaine littérature, elle devrait pouvoir s'appuyer sur I'histoire documentée des lieux dans une confrontation rendant moins aisée la naturalisation et donc l'effacement du sujet. Dans la société urbaine comme dans la fiction, l'hôtel meublé opère le contact et fait du parcours migratoire une épreuve graduée, qui conduit peut-être à la disparition finale, mais dont il constitue une sorte d'étalon et un pôle organisateur.

\section{Références bibliographiques}

Barrère Céline (2009) Placements, déplacements et négociations de l'étranger. Les hôtels meublés de Paris : des espaces de projection et de compensation identitaire, Lieux Communs-LAUA, 12, pp. 33-54.

Barrère Céline et Lévy-Vroelant Claire (2012) Hôtels meublés à Paris. Enquête sur une mémoire de l'immigration, Paris Grâne, Créaphis, 305 p.

Bégout Brice (2003) Lieu commun. Le motel américain, Paris, Allia, 182 p.

Bhabha Homi (2007) Les Lieux de la Culture, Paris, Payot, 416 p.

Bonn Charles (1994) Le voyage innommable et le lieu du dire : Émigration et errance dans l'écriture maghrébine francophone, Revue de Littérature Comparée, 1 (269), pp. 47-59.

Buscatto Marie (2006) Voyage du côté des "perdants " et des "entrepreneurs de mémoire ", Ethnologie française, 36 (4), pp. 745-748.

Céline Louis-Ferdinand (1981 [1952]) Voyage au bout de la nuit, Paris, nrf Gallimard, 505 p.

Chamoiseau Patrick (1997) Écrire en pays dominé, Paris, Gallimard, 316 p.

Faure Alain et Lévy-Vroelant Claire (2007) Une Chambre en ville. Hôtels meublés et garnis à Paris 1860-1990, Paris Grâne, Créaphis, 430 p. 
Foucault Michel (1984) Des espaces autres. Dits et écrits, Architecture, Mouvement, Continuité, 5, pp. 46-49.

Glissant Édouard (1990) Poétique de la relation, Paris, Gallimard, 239 p.

Gotman Anne (2001) Le sens de I'hospitalité. Essai sur les fondements sociaux de l'accueil de l'autre, Paris, Presses Universitaires de France, 507 p.

Halbwachs Maurice (1997 [1925]) La Mémoire collective, Paris, Albin Michel, 295 p.

Halbwachs Maurice (1994 [1925]) Les cadres sociaux de la mémoire, Paris, Albin Michel, $368 \mathrm{p}$.

Halbwachs Maurice (1970 [1938]) La Morphologie sociale, Paris, Armand Colin, $190 \mathrm{p}$.

Harel Simon (2005) Les Passages obligés de l'écriture migrante, Montréal, Éditions XYZ, 250 p.

Hayden Dolores (1994) The power of place. Claiming urban landscape at people's history, Journal of Urban history, 20 (4), pp. 466-485.

Harbi Mohamed (2004) Entre mémoire et histoire : un témoignage sur la politisation de l'immigration maghrébine en France, in Aïssa Kadri et Gérard Prévost Éds., Mémoires algériennes, Paris, Syllepse, pp. 49-57.

Heinich Nathalie et Julien Ténédos (2007) La sociologie à l'épreuve de l'art, Paris, Aux lieux d'être, 125 p.

Hebbert Michael (2005) The street as a locus of collective memory, Environment and Planning: Society and Space, 23, pp. 581-596.

Jankel Stéphanie et Lévy-Vroelant Claire (2007) Les hôtels meublés à Paris. Diagnostic et premier bilan du plan d'action engagé, $51 \mathrm{p}$. [en ligne] consulté le 30/04/2011. URL : http://www.apur.org/etude/hotels-meubles-paris-diagnostic-etpremier-bilan-plan-action-engage

Le Blanc Guillaume (2010) Une voix à soi, De(s)générations, 12, pp. 7-14.

Lévy-Vroelant (2000) Logements de passage. Normes, formes, expériences, Paris, L'harmattan, $298 \mathrm{p}$.

Lopès Henri (1997) Le Lys et le flamboyant, Paris, Seuil, 430 p.

Massey Doreen (1995) Places and their pasts, History workshop journal, 39 (1), pp. 182-192.

Mbembé Achile (2006) Qu'est-ce que la pensée postcoloniale?, Esprit, pp. 117-133.

Michel Andrée (1960) Famille, industrialisation, logement, Population, 15 (4), pp. 727-728.

Modiano Patrick (1997) Dora Bruder, Paris, Gallimard, 145 p.

Modiano Patrick (1990) Voyage de noces, Paris, Gallimard, 157 p.

Noiriel Gérard (2007) À quoi sert l'identité nationale ?, Paris, Agone, 156 p.

Nora Pierre (Dir.) (1997) Les Lieux de mémoire, Paris, Gallimard, 3 tomes, 4751 p. 
Perec Georges (1994) Récits d'Ellis Island, Paris, P.O.L., 71 p.

Perec Georges (1978) Je me souviens, Paris, Hachette, 147 p.

Pinçonnat Crystel (2007) Le Paris maghrébin, une capitale littéraire invisible, in Crystel Pinçonnat et Chantal Liaroutzos Dir., Paris, cartographies littéraires, Paris, Le Manuscrit, pp. 275-303.

Pollak Michael (1993) Une identité blessée : études de sociologie et d'histoire, Paris, Métailié, 415 p.

Riot-Sarcey Michèle (2002) Temps et histoire en débat. "Tout s'oublie " et " rien ne passe ", Revue d'histoire du XIXe siècle, 25, pp. 7-15.

Sayad Abdelmalek (1980) Le foyer des sans famille, Actes de la recherche en sciences sociales, 32 (1), pp. 89-103.

Schérer René (1993) Zeus hospitalier. Éloge de I'hospitalité, Paris, Armand Colin, $199 \mathrm{p}$.

Semprun Jorge (1994) L'Écriture ou la vie, Paris, Gallimard, 400 p.

Spivak Gayatri Chakravorty (1988) Can the Subaltern Speak?, in Cary Nelson and Lawrence Grossberg Eds., Marxism and the Interpretation of Culture, Chicago, University of Illinois Press, pp. 271-313.

Stora Benjamin (2007) La Guerre des mémoires, La Tour d'Aigues, Éditions de l'Aube, $108 \mathrm{p}$.

Veschambres Vincent (2008) Traces et mémoires urbaines. Enjeux sociaux de la patrimonialisation et de la démolition, Presses Universitaires de Rennes, 315 p.

Yong-Sook Lee and Brenda Yeoh S.-A. (2004) Globalisation and the Politics of Forgetting, Urban Studies, 41 (12), pp. 2295-2301. 


\section{Claire Lévy-Vroelant}

\section{... « Comment décrire ? Comment raconter ?... ".. La mémoire perdue des hôtels meublés parisiens}

Les hôtels meublés parisiens semblent les vestiges stigmatisés d'une époque révolue. On les considère ici au contraire comme des milieux de mémoire. L'approche place le sujet au centre en mettant en regard deux modes d'énonciation : les conversations, récits, anecdotes, provenant des logeurs et des logés de vingt hôtels meublés parisiens ; une sélection de textes littéraires d'auteurs francophones exprimant le rapport du narrateur avec l'hôtel. Dans la première partie, la matérialité des hôtels est explorée dans son rapport avec la mémoire : entrées, loge, corridor, café, chambres, caves et greniers portent les récits. Mais l'hôtel - c'est l'objet de la seconde partie-, est aussi une forme sociale hétérodoxe tenant de la famille, de la communauté, du regroupement de hasard, résultant du régime particulier instauré par l'hospitalité. L'activité mémorielle sollicitée n'expose pas une guerre des mémoires, le groupe habitant laisse chacun à sa vérité. Elle montre aussi que clients et hôteliers présentent deux régimes mémoriels distincts. La troisième partie aborde la question de la transmission. Les lieux mêmes disparaissent, emportés par la rénovation urbaine, la mobilité sociale éloigne les héritiers. L'écriture littéraire semble aujourd'hui un vecteur significatif de la transmission d'une mémoire des hôtels.

\section{..." "How to Describe? How to Tell?...". The Parisian Furnished Hotels' Lost Memory}

Furnished hotels in Paris often appear as stigmatized relics of ancient times. In this paper, however, they are seen as milieux of memory, placing the subject in the centre. Two modes of expression are compared: conversations, stories and anecdotes collected in twenty furnished Parisian hotels from lodgers and clients; and a selection of literary texts by Francophone authors expressing the relationship between narrator and hotel. In the first part of the analysis, the materiality of hotels is explored as a seat of memory: stories unfold in entry ways, lodge halls, cafes, rooms, cellars and attics. In the second part, the hotel emerges as an unorthodox social form bringing together family, community and strangers - a random encounter of people resulting from the specific regime of hospitality. In the conversations, it is clear that hosts and guests have two distinct ways of remembering events. Memories are also not construed as collective "war memories"; the group leaves each inhabitant to his or her truth. The third part addresses the question of transmission. The place itself disappears, swept away by urban renewal, whilst social mobility discourages the inheritors from continuing the business. As a consequence, literature is now an important vehicle for passing on the memory of the hotels.

\section{... « ¿Cómo describir? ¿Cómo contar?...". La memoria perdida de los hoteles amueblados parisinos}

Los hoteles amueblados parisinos parecen vestigios estigmatizados de una época pasada. A contrario, se los considera aquí como lugares de memoria. El enfoque pone el súbdito al centro haciendo un paralelo entre dos modos de enunciación: las conversaciones, relatos, anécdotas, procediendo de los que albergan y los alojados de los veinte hoteles amueblados parisinos; así que una selección de textos literarios de autores francófonos expresando la relación del narrador con el hotel. En la primera parte, se explora la materialidad de los hoteles en su relación con la memoria: entradas, portería, corredor, café, habitaciones, sótanos y desvanes, llevan los relatos. Pero el hotel es también una forma social heterodoxa basada en la familia, la comunidad, el reagrupamiento de azar, resultante del régimen especial instaurado por la hospitalidad. La actividad memorial solicitada no expone una guerra de las memorias, el grupo habitante deja cada uno a su propia verdad. Demuestra también que clientes y hoteleros presentan dos regímenes memoriales distintos. La tercera parte trata el tema de la transmisión. Los lugares mismos desaparecen, llevados por la renovación urbana, la movilidad social aleja a los herederos. Hoy, la escritura literaria parece un vector significativo de la transmisión de una memoria de los hoteles. 